\title{
IDENTIFICACIÓN Y REGISTRO DE AMMONITES DEL GÉNERO Raimondiceras Spath, 1924, FORMACIÓN LA HERRADURA, LOCALIDAD MORRO SOLAR, LIMA (VALANGINIANO)
}

\author{
Vera ALLEMAN HAEGHEBAERT \\ Universidad Ricardo Palma \\ Museo de Historia Natural "Vera Alleman Haeghebaert” Fac. de Ciencias Biológicas \\ vera.alleman@urp.edu.pe
}

\section{RESUMEN}

Colección taxonómica de referencia depositada en el Departamento de Paleontología del Museo de Historia Natural "Vera Alleman Haeghebaert" de la Universidad Ricardo Palma. Consiste en la identificación, registro y descripción de treinta y dos ejemplares del género Raimondiceras Spath, 1924, de los cuales doce ejemplares fueron atribuidos a la especie Raimondiceras raimondii (Gabb, 1877), quince a la especie Raimondiceras pflückeri (Lisson, 1904) y los cinco restantes a Raimondiceras sp. El estudio taxonómico inicial se apoyó sobre las publicaciones y fósiles originales accesibles de los autores originales; además se utilizó la clave de identificación de las especies de AMMONOIDEA delGrupoMorroSolar,Valanginiano-Hauteriviano de Lima, Perú (Alleman, 2014).

\section{PALABRAS CLAVE}

Taxonomia, Tipos, Ammonitina, Perisphinctaceae, Valanginiano - Hauteriviano.

\section{IDENTIFICATION AND REGISTER AMMONITES GENUS Raimondiceras Spath, 1924, FORMATION LA HERRADURA, MORRO SOLAR LOCATION, LIMA (VALANGINIAN)}

\section{ABSTRACT}

Taxonomic collection of reference reposited in the Paleontology Department of the Natural History Museum "Vera Alleman Haeghebaert"of the Ricardo Palma University. It consists of the identification, register and the description of thirty-two specimens of the genus Raimondiceras Spath, 1924, of which twelve were attributed to the species Raimondiceras raimondii (Gabb, 1877), fifteen to the species Raimondiceras pflückeri (Lisson, 1904) and the remaining five to Raimondiceras sp. The initial taxonomic study was based on the original publications and fossils accessibles to the original authors; In addition, the identification key of the AMMONOIDEA species of the Morro Solar Group, Valanginian-Hauterivian of Lima, Peru (Alleman, 2014) was used. 


\section{KEY WORDS}

Taxonomy, Tipes, Ammonitina, Perisphinctaceae, Valanginian - Hauterivian.

Recibido: 25/03/2019

Aprobado: 22/05/2019

\section{INTRODUCCIÓN}

as especies Raimondiceras raimondii (Gabb, 1877) y Raimondiceras pflückeri (Lisson, 1904) así como el género Raimondiceras Spath, 1924 son tres taxones peruanos. El primero fue un ammonite encontrado por Raimondi A. en el Morro Solar y descrito por W. Gabb en 1877, quien lo ha dedicado a Raimondi.

Más tarde C. Lissón describió dos ejemplares más de la misma especie en 1904 y en 1907. Al mismo tiempo C. Lisson describió también en 1904, dos ejemplares de otra especie nueva de ammonite, dedicándola al Ing. Pflücker, la actual Raimondiceras pflückeri (Lisson, 1904). En 1924 Spath, haciendo una revisión de ammonites a nivel mundial dio existencia al nuevo género Raimondiceras llevando Raimondiceras raimondii a nivel degenotipo.

Resulta que la existencia de estos tres taxones reposa sobre las descripciones y figuraciones de los seis ejemplares originales guardados en las colecciones de Carlos Lisson, en el Museo de Mineralogía y Gabinete de Paleontología de la Universidad Nacional delngeniería,

Actualmente se pueda ubicar dos ejemplares originales de Raimondiceras pflückeri, pero ningún ejemplar original de Raimondiceras raimondii. Adjuntamos que las descripciones e ilustraciones de esas épocas están consideradas ahora bastante obsoletas e incompletas y que se necesita consolidar la validez de estas especies, en la medida de lo posible, por un estudio taxonómico actualizado respaldado por un mayor número de ejemplares, una terminología descriptiva más precisa y el apoyo de la tecnología ilustrativa e reconstructiva del siglo XX.

El objetivo del presente trabajo es la elaboración de una nueva colección registrada de Raimondiceras raimondii (Gabb, 1877) y de Raimondiceras pflückeri (Lissón, 1904), recolectadas en las localidades originales de ambas, considerando eso como un primer avance de un estudio taxonómico actualizado de estas dos especies.

\section{MATERIAL Y MÉTODOS}

El material proviene de la localidad Morro Solar, Departamento de Lima, Provincia de Lima, Distrito de Chorrillos. Contexto litoestratigráfico Grupo Morro Solar, Formación La Herradura, Miembro La Herradura y Miembro La Virgen. Contexto cronoestratigráficoMesozoico,Cretáceo,Valanginiano(Palaciosetd1992).

La recolección se hizo durante las prácticas de campo de las asignaturas de Paleontología y de Paleobiología de los alumnos de la Facultad de Ciencias Biológicas de la Universidad RicardoPalma. 
Nuestra colección dispone de una cantidad de treinta y dos ejemplares, registrados bajo los códigos siguientes: VA010413, VA010513, VA011116, VA020413, VA021116, VA021216 (VE08375), VA030413, VA031116, VA031216 (VE08316(4)), VA041216 (VE08316(3)), VA050413, VA051216, VA060413, VA061216, VA070413, VA071216, VA080413, VA081216 (VE08375)), VA091216 (VE08316(5)), VA101216 (VE08325)), VA110413, VA111216 (VE08323)), VA210413, VA340413(MHNURP-28), VA350413 (MHNURP27), VA381116, VA391116, VA401116, VA450413, VA500413, VA620413, VA720413 (MHNURP-12a)).

El estudio taxonómico inicial se apoyó sobre las publicaciones deW.Gabb (1877) y de C. Lissón (1904, 1907); además se utilizó la clave de identificación de las especies de AMMONOIDEA del Grupo Morro Solar, Valanginiano-Hauteriviano de Lima, Perú (Alleman, 2014).

La identificación taxonómica y el registro han sido cumplidos según las normas exigidas por el Ministerio Nacional de Cultura para su incorporación como bienes muebles de la Nación.

\title{
RESULTADOS
}

A parte que presentamos los ejemplares agrupados según su procedencia de los Miembros La Virgen y La Herradura, todos los ejemplares tienen como registro común los datos siguientes:

Localidad: Morro Solar, Lima, Departamento de Lima, Provincia de Lima, Distrito de Chorrillos. 12 grados $11^{\prime} 05^{\prime \prime}$; 77 grados 01'50"0 (Romero y otros, 1995).

Contexto litoestratigráfico: Grupo Morro Solar, Formación La Herradura (Palacios et al, 1992).

Contexto cronoestratigráfico: Mesozoico, Cretáceo, Valanginiano (Palacios et al, 1992).

Colector: Vera Alleman y alumnos. Identificado por: Vera Alleman.

\author{
PARTE TAXONÓMICA \\ ANIMALIA (según Moore, 1996) \\ Phylum MOLLUSCA \\ Clase CEPHALOPODA Leach, 1817 \\ Orden AMMONOIDEA Zittel, 1884 \\ Suborden AMMONITINA Hyatt, 1889 \\ Superfamilia PERISPHINCTACEAE Steinmann, 1890 \\ Familia NEOCOMITIDAE Salfeld, 1921 \\ Subfamilia NEOCOMITINAE Salfeld,1921 \\ Género Raimondiceras Spath, 1924
}




\section{A. Ejemplares de Raimondiceras raimondii (Gabb, 1877).}

\section{Contexto litoestratigráfico: Grupo Morro Solar, Formación La Herradura, Miembro La Herradura.}

VA011116: Lám. 1. Fig. 1.

Descripción: Impresión de dos vueltas completas y aproximadamente una cuarta parte de la circunferencia de una tercera vuelta; no se observa el dorso. Diámetro del ombligo: veinticinco $\mathrm{mm}$; quince costillas simples sobre la mitad de la penúltima vuelta. Tercera vuelta con ocho costillas visibles de las cuales cuatro costillas Ilevan un tubérculo a nivel de la mitad de la altura del flanco; las costillas tuberculadas están separadas entre sí por una costilla umbilical simple y una costilla intercalada; doce costillas sobre el hombro dorsal. Altura máxima del flanco de la tercera vuelta: veinticinco $\mathrm{mm}$.

Dimensiones y peso de la muestra: longitud máxima: $90 \mathrm{~mm}$; peso: $69 \mathrm{~g}$.

Estado de conservación: Integridad incompleta.

Conservación: Fosilización por impresión, buena.

VA021216 (VE08375): Lám. 1. Fig. 4.

Descripción: Dos partes de una vuelta externa de aproximadamente cuarenta y cinco $\mathrm{mm}$ de largo (reconstitución por pegamento). Ombligo y hombro umbilical no conservados. Altura máxima de la vuelta: mayor a veinte y cinco $\mathrm{mm}$. Una sola costilla tuberculada conservada, la cual lleva el tubérculo a la altura de la mitad del flanco; de ambos lados, sobre el dorso, desembocan diez y seis costillas, entre simples e intercaladas, formando una figura en "chevron" (Lissón, 1907: Lam. 5, fig. 1b); dorso confajamedianacasiimperceptible(Fig.7).Cortetransversaldeforma trapezoidal(Fig. 8): estimado a veinte $\mathrm{mm}$ de ancho a la base y diez $\mathrm{mm}$ de ancho a nivel del supuesto hombrodorsal.

Dimensiones y peso de la muestra: Longitud máxima: $45 \mathrm{~mm}$; peso: $38 \mathrm{~g}$.

Estado de conservación: Integridad incompleta.

Conservación: Fosilización por petrificación, buena.

\section{VA030413.}

Descripción: Fragmento de una vuelta de treinta y cinco $\mathrm{mm}$ de largo. Parte del ombligo presente pero demasiado mal conservado para poder dar mayor información; no se distingue el límite del dorso. Flanco de veinte y siete $\mathrm{mm}$ de altura máxima. Cinco costillas umbilicales de las cuales tres llevan un tubérculo a mitad dela altura del flanco; las costillas tuberculadas están separadas por una costilla umbilical simple y por una costilla intercalada, separadas también por una intercostilla amplia. No se observan tubérculos umbilicales; quince costillas desembocansobreelhombro deldorsoformandoun"chevron"deánguloagudo.

Dimensiones y peso de la muestra: longitud máx.: $53 \mathrm{~mm}$; peso: $35 \mathrm{~g}$.

Estado de conservación: Integridad incompleta.

Conservación: Fosilización por impresión, buena. 


\section{VA060413.}

Descripción: Fragmento incompleto de casi media vuelta de un ejemplar de un diámetro mayor a setenta $\mathrm{mm}$. No se observan las estructuras a nivel del ombligo. Altura máxima del flanco: treinta $\mathrm{mm}$; doce costillas umbilicales de las cuales tres son tuberculadas y forman una hilera a la altura de la mitad del flanco; a las costillas umbilicadas se juntan una cantidad complementaria de costillas intercaladas sobre el dorso donde se observan nítidamente veinte costillas. Dorso mal conservado con estrecha faja lisamediana.

Dimensiones y peso de la muestra: Longitud máxima: 90 mm; peso: $64 \mathrm{~g}$.

Estado de conservación: Integridad incompleta.

Conservación: Fosilización por impresión, buena.

\section{VA070413.}

Descripción: El ejemplar consiste en un fragmento incompleto de casi la mitad de una vuelta; diámetro: un poco más de ochenta mm; altura máxima del flanco: superior a veinte y cinco $\mathrm{mm}$; no se distinguen los límites del dorso y de la región umbilical. Diez y siete costillas umbilicales de las cuales cinco llevan un tubérculo a mitad de la altura del flanco, las costillas tuberculadas están separadas por tres costillas simples.

Dimensiones y peso de la muestra: Longitud máxima: $125 \mathrm{~mm}$; peso: $320 \mathrm{~g}$.

Estado de conservación: Integridad incompleta.

Conservación: Fosilización por impresión, buena.

\section{VA071216.}

Descripción: Impresión de una última vuelta incompleta de setenta $\mathrm{mm}$ de largo; espesor mayor a veinte $\mathrm{mm}$; altura máxima del flanco: superior a veinte $\mathrm{mm}$; trece costillas umbilicales; hilera de tres costillas que llevan un tubérculo a mitad de la altura del flanco; las costillas tuberculadas están separadas por dos costillas umbilicales simples y una costilla dorsal intercalada; no se observan tubérculos umbilicales; veintiuna costillas llegan al dorso. Dorso con las costillas dispuestas en forma "chevron"y separadas con casi un imperceptible espacio mediano liso. El ombligo no está conservado.

Dimensiones y peso de la muestra: Longitud máxima: $100 \mathrm{~mm}$; peso: $73 \mathrm{~g}$.

Estado de conservación: Integridad incompleta.

Conservación: Fosilización por impresión, buena.

VA081216 (VE08375): Lám. 1. Fig. 3.

Descripción: Fragmento incompleto de una vuelta interna de cuarenta y cinco $\mathrm{mm}$ de largo; altura máxima del flanco: treinta $\mathrm{mm}$; doce costillas umbilicales de las cuales tres se bifurcan formando un tubérculo que se observan en conjunto como una hilera de tres tubérculos a la altura de la mitad del flanco; dorso con quince costillas. Dorso hundido con faja lisa mediana. Corte transversal trapezoide; base: diez $\mathrm{mm}$ de ancho; dorso: seis $\mathrm{mm}$ de ancho; altura: dieciocho $\mathrm{mm}$. 
Dimensiones y peso de la muestra: Longitud máxima: $50 \mathrm{~mm}$; peso: $29 \mathrm{~g}$.

Estado de conservación: Integridad incompleta.

Conservación: Fosilización por impresión, buena.

VA110413: Lám. 1. Fig. 2.

Descripción: El ejemplar consiste de una porción de vuelta de cuarenta mmde largo; flanco de treinta mm de alto; dos costillas umbilicales engrosadas sobre el hombro umbilical; ocho costillas umbilicales de las cuales dos se bifurcan a partir de un nudo ubicado a mitad de la altura de la vuelta; la intercostilla de las dos costillas tuberculadas presenta dos costillas simplesy una intercalada a partir del dorso.

Dimensiones y peso de la muestra: Longitud máxima: $100 \mathrm{~mm}$; peso: $315 \mathrm{~g}$.

Estado de conservación: Integridad incompleta.

Conservación: Fosilización por impresión, buena.

VA340413 (MHNURP-28).

Descripción: Casi la mitad de una última vuelta de un ejemplar deformado; diámetro mayor a cincuenta y cinco $\mathrm{mm}$; diámetro del ombligo mayor a veinte $\mathrm{mm}$; altura máxima del flanco veinte y tres $\mathrm{mm}$; hilera de tubérculos laterales, poco elevados; costillas umbilicales engrosadas sobre el hombro umbilical; veinte y cinco costillas sobre el hombro del dorso; dorso no conservado.

Dimensiones y peso de la muestra: longitud máxima: 120 mm; peso: $365 \mathrm{~g}$.

Estado de conservación: Integridad incompleta.

Conservación: Fosilización por impresión, regular.

\section{VA450413.}

Descripción: Casi la mitad de un ejemplar deformado; diámetro de ejemplar mayor a cincuenta y cinco $\mathrm{mm}$; diámetro del ombligo alrededor de quince $\mathrm{mm}$; altura máxima de la vuelta veinte $\mathrm{mm}$; tubérculos laterales poco elevados, ausencia de tubérculos periumbilicales; varias costillas separan las costillas tuberculadas; dorso con faja medianalisa.

Dimensiones y peso de la muestra: Longitud máxima: $170 \mathrm{~mm}$; peso: $859 \mathrm{~g}$.

Estado de conservación: Integridad incompleta.

Conservación: Fosilización por impresión, buena.

\section{VA500413.}

Descripción: Casi la cuarta parte de un ejemplar completo; mitad del diámetro de la concha: treinta y cinco $\mathrm{mm}$; altura máxima de la vuelta externa: veinte y cinco $\mathrm{mm}$; siete costillas periumbilicales mal conservadas; no se aprecian tubérculos periumbilicales; tubérculos sobre el flanco mal conservados; varias costillas separan las costillas tuberculadas; trece costillas sobre el hombro del dorso. Dorso con faja mediana lisa. Ombligo con tres vueltas; mitad del diámetro del ombligo: alrededor de doce $\mathrm{mm}$. Penúltima vuelta completamente conservada, con una parte parcialmente incompleta dejando apreciar que la última vuelta recubre más de la mitad del flanco de la vuelta anterior y oculta los pequeños tubérculos ubicados sobre el flanco en la 
bifurcación de las costillas tuberculadas; una costilla simple separa las dos costillas tuberculadas; altura máxima de la penúltimavuelta, incompletamentevisible:nueve $\mathrm{mm}$; altura de la penúltima vuelta cubierta: tres $\mathrm{mm}$; penúltima vuelta con veinte y siete costillas umbilicales de las cuales tres están observables completamente.

Dimensiones y peso de la muestra: Longitud máxima: $220 \mathrm{~mm}$; peso: $174 \mathrm{~g}$. Estado de conservación: Integridad incompleta.

Conservación: Fosilización por impresión, buena.

VA720413 (MHNRP-12a).

Descripción: El ejemplar consiste en un fragmento incompleto de una vuelta de sesenta y seis $\mathrm{mm}$ de largo; altura máxima del flanco: treinta y cinco $\mathrm{mm}$; costillas umbilicales engrosadas sobre el hombro umbilical; una hilera de cuatrotubérculos a laaltura dela mitad delflanco; dorso con diezy siete costillasy trazas decostillas.

Dimensiones y peso de la muestra: Longitud máxima: $130 \mathrm{~mm}$; peso: $371 \mathrm{~g}$. Estado de conservación: Integridad incompleta, mala.

Conservación: Fosilización por impresión, buena.

\section{B. Ejemplares de Raimondiceras pflückeri (Lisson, 1904)}

\section{Contexto litoestratigráfico: Grupo Morro Solar, Formación La Herradura, Miembro La Herradura.}

\section{VA010413.}

Descripción: Parte de una vuelta de cuarenta y siete $\mathrm{mm}$ de largo; altura máxima de la vuelta mayor a veinte y cinco mm; tres costillas tuberculadas a la altura de la mitad del flanco; hombro umbilical no conservado; costillas tuberculadas separadas por espacios amplios con costillas simples e intercaladas; diez y seis costillas desembocan sobre el dorso; dorso con estrecha faja mediana lisa. Ombligo no conservado.

Se le atribuye a la especie pflückeri por la forma puntiaguda de los nudos y el ángulo agudo de la disposición de las costillas en forma de"chevron"sobre el dorso.

Dimensiones y peso de la muestra: longitud máxima $110 \mathrm{~mm}$; peso: $184 \mathrm{~g}$.

Estado de conservación: Integridad incompleta.

Conservación: Fosilización por impresión, buena.

\section{VA020413.}

Descripción: Impresión de setenta $\mathrm{mm}$ de una vuelta incompleta cuyo dorso no está conservado; ombligo no conservado. La altura máxima del flanco es mayor a treinta y cinco $\mathrm{mm}$; dos costillas bituberculadas visibles espaciadas entre sí por una intercostilla amplia en la cual se localizan dos costillas intercaladas.

Dimensiones y peso de la muestra: Longitud máxima: $130 \mathrm{~mm}$; peso: $191 \mathrm{~g}$.

Estado de conservación: Integridad incompleta.

Conservación: Fosilización por impresión, buena. 


\section{VA021116.}

Descripción: Impresión de un ejemplar incompleto: ombligo casi completo y un estimado de una cuarta parte de la circunferencia de la tercera vuelta; diámetro mayor a ochenta $\mathrm{mm}$. Diámetro del ombligo: mayor a cuarenta $\mathrm{mm}$. Altura del flanco de la vuelta externa: mayor a treinta y cinco $\mathrm{mm}$; Penúltima vuelta con treinta y tres costillas periumbilicales de las cuales solamente se conservaron tres costillas bituberculadas; tubérculos periumbilicales alargados y poco pronunciados: costillas simples están ubicadas entre las costillas tuberculadas sobre el flanco, separadas entre sí por intercostillas amplias. Dorso no conservado.

Dimensiones y peso de la muestra: Longitud máxima: $240 \mathrm{~mm}$; peso: $2050 \mathrm{~g}$. Estado de conservación: Integridad incompleta.

Conservación: Fosilización por impresión, buena.

\section{VA050413.}

Descripción: Impresión de casi la mitad de una vuelta externa de un ejemplar con un diámetro estimado de aproximativamente ochenta $\mathrm{mm}$. Medio ombligo delimitado, pero no conservado; diámetro del ombligo: treinta mm. Altura máxima del flanco: treinta y cinco $\mathrm{mm}$; diez y siete costillas umbilicales de las cuales tres son bituberculadas sobre la mitad de la altura del flanco y llevan tubérculos periumbilicales bien redondeadas. Dorso con veinte y seis costillas estiradas hacia adelante formando un dibujo en forma de"chevron"con ángulo agudo.

Dimensiones y peso de la muestra: Longitud máxima: $130 \mathrm{~mm}$; peso: $626 \mathrm{~g}$.

Estado de conservación: Integridad incompleta.

Conservación: Fosilización por impresión, buena.

\section{VA080413.}

Descripción: El ejemplar consiste en una porción de vuelta de cuarenta mm de largoy treinta $\mathrm{mm}$ de altura. Ocho costillas umbilicales de las cuales dos son bituberculadas

Dimensiones y peso de la muestra: Longitud máxima: $140 \mathrm{~mm}$; peso: $283 \mathrm{~g}$.

Estado de conservación: Integridad incompleta.

Conservación: Fosilización por impresión, buena

\section{VA381116.}

Descripción:Aproximativamentela mitad de una vuelta externay una partedistal dela penúltima vuelta del moldeexterno de unejemplaradulto, algo deformado; diámetro: sesenta y seis $\mathrm{mm}$. Penúltima vuelta tuberculada, parcialmente presente pero mal conservada. Altura máxima del flanco de la vuelta externa: treinta $\mathrm{mm}$; dos hileras de tubérculos sobre el flanco: una umbilical y otra la altura de la mitad del flanco; diez y siete costillas visibles sobre el hombro dorsal derecho y doce sobre el izquierdo. Sección transversal (fig. 36) de la concha de forma achatada, trapezoidal: altura del flanco: treinta $\mathrm{mm}$, ancho: veinte $\mathrm{mm}$. Dorso surcado con veinte y cuatro costillas dirigidas adelante y dispuestas en forma de "chevron". 
Dimensiones y peso de la muestra: Longitud máxima: 70 mm; peso: $102 \mathrm{~g}$.

Estado de conservación: integridad incompleta.

Conservación: Fosilización por impresión, buena.

\section{Ejemplares de Raimondiceras pflückeri (Lisson, 1904)}

\section{Contexto litoestratigráfico: Grupo Morro Solar, Formación La Herradura, Miembro La Virgen.}

\section{VA010513.}

Descripción:Veintisiete mm de la vuelta externa y la mitad de la penúltima vuelta de un ejemplar juvenil incompleto; centro del ombligo ausente. Altura de la vuelta externa: diez $\mathrm{mm}$; altura de la penúltima vuelta: dos $\mathrm{mm}$. Seis costillas tuberculadas separadas por costillas intercaladas en la última vuelta. Siete costillas en la penúltima vuelta.

Dimensiones y peso de la muestra: Longitud máxima: $10 \mathrm{~mm}$; peso: $1 \mathrm{~g}$.

Estado de conservación: Integridad incompleta.

Conservación: Fosilización por impresión, regular, ejemplar frágil.

as. Vuelta anterior con 7 costillas tuberculadas visibles.

VA031116 (VA08318(1)): Lám. 1. Fig. 7.

Descripción: Impresión de un ejemplar adulto conservando la vuelta externa casi completa; diámetro superior a ciento y treinta $\mathrm{mm}$; altura máxima del flanco: mayor a cincuenta $\mathrm{mm}$.; Ombligo delimitado, sin estructuras conservadas; diámetro del ombligo sesenta $\mathrm{mm}$. Once costillas umbilicales reducidas a tubérculos alargadas perdiendo su relieve progresivamente a partir de la mitad de la altura del flanco a lo largo del crecimiento del animal. Dorso truncado (fig. 11), liso, donde se aprecian ondulaciones superficiales dirigidas hacia adelante. Sección transversal incompleta.

Observación: Las medidas del ejemplar están comparables con las mencionadas por Lissón, 1907, tabla p. 38, y el dorso descrito por Lissón 1907, pág. 39.

Dimensiones y peso de la muestra: Longitud máxima: $130 \mathrm{~mm}$; peso: $1545 \mathrm{~g}$.

Estado de conservación: Integridad incompleta.

Conservación: Fosilización por impresión, buena.

VA031216 (VE08316(4)).

Descripción: Ejemplar aplastado presentando tres vueltas incompletas. Altura de la vuelta más externa: mayor a treinta $\mathrm{mm}$; altura de la vuelta anterior:quince mm; altura de la vuelta más interna: siete $\mathrm{mm}$. Las costillas umbilicales bituberculadas Ilevan tubérculos puntiagudos a nivel del contacto de las vueltas anteriores.

Observación: Ejemplar comparable con la fig. No 3, Lám. V, Lissón ,1907.

Dimensiones y peso de la muestra: longitud máx.: $60 \mathrm{~mm}$; peso: $38 \mathrm{~g}$.

Estado de conservación: Integridad incompleta.

Conservación: Fosilización por petrificación, buena. 
VA041216 (VE08316(3)).

Descripción: Impresión de un ejemplar aplastado, casi completa, de cincuenta y cinco $\mathrm{mm}$ de diámetro; ombligo de un apreciado de veinte y dos $\mathrm{mm}$ de diámetro; altura del flanco de la vuelta externa: superior a veinte y cinco $\mathrm{mm}$; dos hileras de tubérculos puntiagudos: una a nivel de la mitad de la altura del flanco y otra periumbilical. No se perciben bien las costillas por estar mal conservada la muestra. Ornamentación del dorso noconservada.

Observación: Es una forma joven del mismo diámetro del ejemplar figurado por Lissón, 1907, p. XXXVIII.

Dimensiones y peso de la muestra: longitud máx.: $56 \mathrm{~mm}$; peso: $28 \mathrm{~g}$.

Estado de conservación: Integridad incompleta.

Conservación: Fosilización por impresión, no muy buena.

\section{VA051216.}

Descripción: Cuarenta $\mathrm{mm}$ de una vuelta de un ejemplar juvenil. Ombligo no conservado. Altura máxima del flanco: diez y ocho $\mathrm{mm}$. Una hilera de dos tubérculos puntiagudos a nivel de la mitad de la altura del flanco. No se puede apreciar el borde umbilical. Ornamentación del dorso no conservada.

Observación: Ejemplar comparable con la descripción de Lissón, 1904; p. 61 y también con la ilustración en Lissón, 1907, fig. No 6, lám. 4.

Dimensiones y peso de la muestra: Longitud máxima: $40 \mathrm{~mm}$; peso: $6 \mathrm{~g}$.

Estado de conservación: Integridad incompleta.

Conservación: Fosilización por petrificación, regular, frágil.

VA061216: Lám 1. Fig. 6

Descripción: Impresión de un ejemplar casi completa de un diámetro mayor a ciento y quince $\mathrm{mm}$; ombligo de cuarenta $\mathrm{mm}$ de diámetro. Altura máxima del flanco cincuenta $\mathrm{mm}$; costillas periumbilicales cuya hilera de tubérculos se van progresivamentealargando en costillasespesas, separadas porintercostillasamplias y luego, a lo largo del crecimiento del individuo, terminan formando pliegues amplios. Dorso con faja lisa. Espesor máximo apreciable a nivel de la porción proximal de la concha: treinta $\mathrm{mm}$.

Observación: Ejemplar con dimensiones comparables al ejemplar mencionado en la tabla p. XXX VIII, Lissón, 1907.

Dimensiones y peso de la muestra: longitud máxima: $140 \mathrm{~mm}$; peso: $366 \mathrm{~g}$.

Estado de conservación: Integridad incompleta, en vía de destrozarse.

Conservación: Fosilización por impresión, mala.

VA091216 (VE08316 (5)) (16) Lám. 1. Figs. 8, 9.

Descripción: El ejemplar consiste de sesenta $\mathrm{mm}$ de una vuelta de concha bastante gastada; altura del flanco: veinte $\mathrm{mm}$; de la presencia de quince costillas periumbilicales 
sola se conservaron una costilla bituberculada y una hilera de dos tubérculos laterales; dorso con diez y nueve costillas dispuestas en forma de "chevron" con ángulo amplio. Corte transversal proximal de la concha: dorso achatado; diámetro a nivel del dorso: cinco $\mathrm{mm}$, diámetroa la base delavuelta: diez $\mathrm{mm}$, altura del flanco:quince mm.

Dimensiones y peso de la muestra: Longitud máxima: $60 \mathrm{~mm}$; peso: $31 \mathrm{~g}$. Estado de conservación: Integridad incompleta.

Conservación: Fosilización por petrificación, buena.

VA391116: Lám. 1. Fig. 5.

Descripción: Fósil petrificado de un individuo juvenil: treinta y ocho $\mathrm{mm}$ de una vuelta externa con penúltima vuelta adjunta. Sobre la penúltima vuelta se observan dos costillas cuyos tubérculos, que corresponden a los tubérculos ubicados a la mitad de la altura del flanco, han sido conservados. Altura del flanco de la última vuelta: diez y siete $\mathrm{mm}$; ancho: trece $\mathrm{mm}$. Tres costillas periumbilicales bituberculadas; las costillas periumbilicales están separadas por una costilla intercalada; once costillas sobre el hombro dorsal. Dorso surcado con once costillas dirigidas adelante y dispuestas en forma de"chevron"Secciones transversales: sección transversal distal de la vuelta: dorso redondeado, achatado, Sección transversal proximal de la vuelta: dorso achatado forma trapezoidal:ocho mm; altura del flanco:quince $\mathrm{mm}$.

Dimensiones y peso de la muestra: Longitud máxima: $38 \mathrm{~mm}$; peso: $15 \mathrm{~g}$.

Estado de conservación: integridad incompleta.

Conservación: Fosilización por petrificación, regular.

\section{VA401116.}

Descripción: Molde parcialmente petrificado, incompleto, de ciento y quince mm de la última vuelta de un ejemplar senil (acomparar con el ejemplar en Lissón 1907, p.39); ombligo ausente. Tres costillas periumbilicales radiales, rectas y cuya elevación redondeada se ha transformado en ondulaciones para terminar a desaparecer más o menos a nivel de la mitad de la altura del flanco. Dorso marcado por reminiscencias de algunas costillas, marcadas como gruesos pliegues. Sección transversal proximal de la vuelta: forma abovedada, altura de la vuelta: cincuenta y cinco $\mathrm{mm}$; ancho de la vuelta:treintamm.

Dimensiones y peso de la muestra: Longitud máxima: $115 \mathrm{~mm}$; peso: $348 \mathrm{~g}$.

Estado de conservación: integridad incompleta.

Conservación: Fosilización por petrificación, buena.

\section{Ejemplares de Raimondiceras sp. Spath, 1924.}

Contexto litoestratigráfico: Grupo Morro Solar, Formación La Herradura, Miembro La Herradura.

\section{VA210413.}

Descripción: Parte de una mitad superior de una vuelta externa de cincuenta cinco 
mm de largo; hombro umbilical y ombligo no conservados. Altura máxima observable del flanco: mayor a diez y ocho $\mathrm{mm}$; veinte costillas desembocan sobre el dorso de las cuales solamente en una se puede observar un tubérculo probablemente ubicado a la altura de la mitad del flanco; dorso con faja mediana lisa.

Dimensiones y peso de la muestra: Longitud máxima: $100 \mathrm{~mm}$; peso: $218 \mathrm{~g}$.

Estado de conservación: Integridad incompleta.

Conservación: Fosilización por impresión, buena.

\section{VA350413.}

Descripción:Impresión de una parte distal de una vuelta externa de treintay cinco mm de largo; dorso y hombro umbilical no conservados; altura máxima del flanco mayor a veinte y cinco $\mathrm{mm}$. Sobre el hombro desembocan ocho costillas de las cuales tres llevan un tubérculo sobre la mitad de la altura del flanco.

Dimensiones y peso de la muestra: Longitud máxima: 75 mm; peso: $158 \mathrm{~g}$.

Estado de conservación: Integridad incompleta.

Conservación: Fosilización por impresión, buena.

\section{VA620413.}

Descripción: Impresión de sesenta mm de largo de la mitad superior de una vuelta probablemente exterior de un ejemplar adulto incompleto. Mitad inferior de la vuelta y ombligo ausentes Altura visible de la media vuelta mayor a $45 \mathrm{~mm}$. Trece costillas desembocan sobre el hombro dorsal estirándose hacia adelante formando undorso confaja central lisa:trestubérculos visiblesa nivel dela mitad delaaltura de la vuelta.

Dimensiones y peso de la muestra: Longitud máxima: $140 \mathrm{~mm}$; peso: $851 \mathrm{~g}$.

Estado de conservación: integridad incompleta.

Conservación: Fosilización por impresión, buena.

\section{E. Ejemplares de Raimondiceras sp. Spath, 1924.}

\section{Contexto litoestratigráfico: Grupo Morro Solar, Formación La Herradura, Miembro La Virgen.}

$$
\text { VA101216 (VE08325). }
$$

Descripción: Cincuenta y cinco $\mathrm{mm}$ de una probable vuelta externa conservando solamente la mitad inferior del flanco. Tres costillas periumbilicales radiales amplias, evaneciéndose mientras que las intercostillas se amplifican.

Dimensiones y peso de la muestra: Longitud máxima: $120 \mathrm{~mm}$; peso: $530 \mathrm{~g}$.

Estado de conservación: Integridad incompleta.

Conservación: Fosilización por impresión, buena. 
VA111216 (VE08323).

Descripción: Contramolde del ejemplarVA101216. Cincuenta mm de la contraparte positiva de la mitad inferior del flanco; tres costillas periumbilicales radiales evaneciéndose mientras que las intercostillas se amplifican.

Dimensiones y peso de la muestra: Longitud máxima: $93 \mathrm{~mm}$; peso: $86 \mathrm{~g}$.

Estado de conservación: Integridad incompleta.

Conservación: Fosilización por impresión, buena.

\section{CONCLUSIONES}

Se ha precisado el lugar donde A. Raimondi y C. Lissón consiguieron los primeros ejemplares de Raimondiceras raimondii y Raimondiceras pflückeri: la Formación La Herradura que aflora en los cerros del Morro Solar de Chorrillos. Raimondiceras pflückeri se recolectó tanto en el Miembro La Virgen como en el Miembro La Herradura. R.raimondiiseencontró únicamenteen el Miembro La Herradura.

Nuestra contribución consiste en la presentación de la existencia de una nueva colección registrada de Raimondiceras raimondii (Gabb, 1877) y Raimondiceras pflückeri recolectada en la localidad original y depositada en el Museo de Historia Natural"Vera Alleman Haeghebaert"de la Universidad Ricardo Palma.

Es un primer avance de un estudio taxonómico actualizado de la especie y hacia la realización de una nueva colección de material como base de reconstrucción de una nueva colección de tipos de ambas especies.

La colección se podría complementar con una mayor cantidad de individuos, entre otros, con ejemplares de los afloramientos de la Formación La Herradura ubicados en la Isla SanLorenzo.

Se espera que la presente colección debidamente investigada ratifique y complete los datos de los autores anteriores y amplifique los conocimientos paleobiológicos de estas dos especies.

Recomendamos este material como objeto de tesis de grado para un estudio profundizado de las dos especies de Raimondiceras.

\section{AGRADECIMIENTOS}

Esta investigación fue aprobada por Acuerdo de Consejo Universitario No 14682016, por lo cual agradecemos la Universidad Ricardo Palma, así como al Instituto Peruano de Astronomía por el apoyo al presente tema de investigación. 


\section{REFERENCIAS}

GABB, W. (1877). Description of a Collection of fossils made by Dr. A. Raimondi in Perú. Journal of the Academy of Natural Science Philadelphia, Serie 2. 8: 263-336.

Lisson, C. (1904). Los Tigillites del Salto del Fraile y algunos Sonneratias del Morro Solar. Contribución a la Geología de Lima y Alrededores. Imprenta Gil.

Lisson, C. (1907). Contribución a la Geología de Lima y Alrededores. Imprenta Gil.

Palacios, O., Caldos, J. y V. Churchil . (1992). Geología de los Cuadrángulos de Lima, Lurín, Chancay y Chosica. Boletín No 43, Serie A. Instituto Geológico Minero y Metalúrgico: $152 \mathrm{pp}$.

Rivera, R.y V.Alleman. (1974). Fósiles"Tipos"conservadosen el Perú. Boletín de la SociedadGeológica del Perú, 44: 80-104.

Romero, L., Aldana,M., Villavicencia,E. y J.Ramiez.1995.FaunayFlorafósildelPerú. Bol. 17, Serie D: Estudios Especiales. Instituto Geológico Minero y Metalúrgico

Tapia, P. (1988). Revisión bibliográfica de los Ammonoidea (Cephalopoda, Mollusca) del Cretáceo de Lima. Tesis Bach. Biología, Lima: Universidad Ricardo Palma.

Wright, C., Callomon, J. y M. Howarth en Moore. R. 1996. Treatise on Invertebrate Paleontology, part L, MOLLUSCA 4. Geological Society of America, University of Kansas Press. 


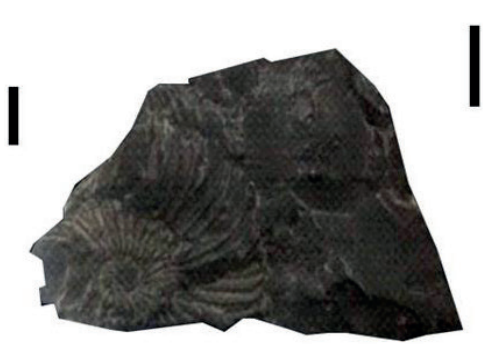

Fig 1. VA011116 Raimondiceras raimondii (Gabb, 1877).

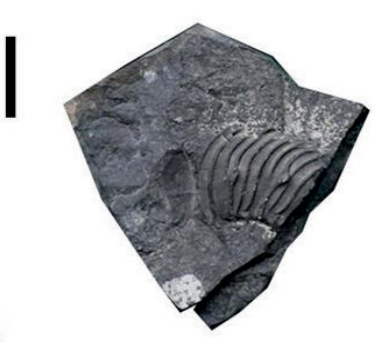

Fig. 2. VA110413

Raimondiceras raimondii (Gabb, 1877)

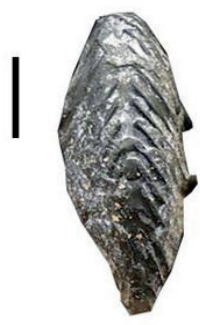

Fig. 3. VA081216 Raimondiceras raimondii (Gabb, 1877). Dorso.

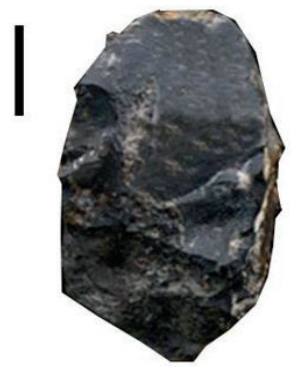

Fig. 4.VA021216

Raimondiceras raimondii

(Gabb, 1877).

Corte transversal

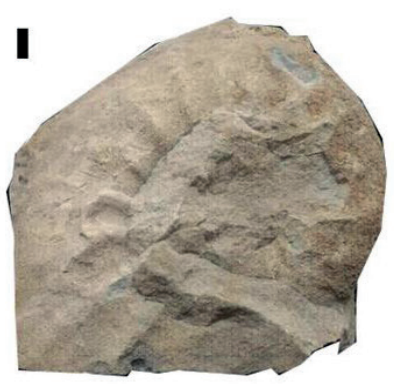

Fig. 7. VA031116

Raimondiceras pflücken

(Lissón, 1904)).

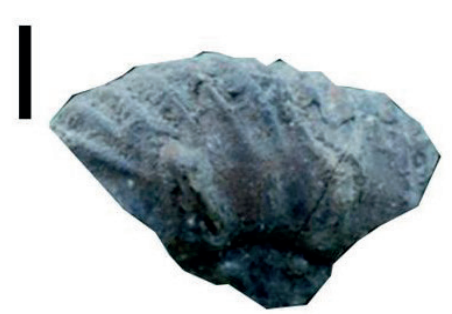

Fig. 5. VA391116.

Raimondiceras pflücker $i$ (Lissón, 1904).

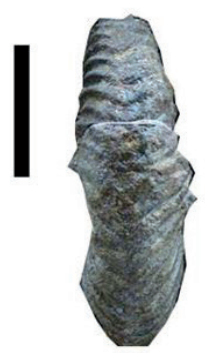

Fig. 8. VA091216.

Raimondiceras pflückeri (Lissón, 1904). Dorso

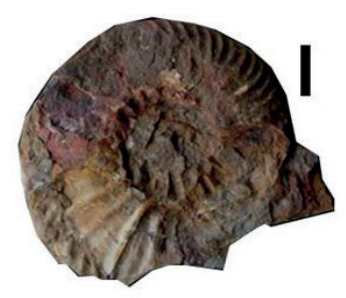

Fig. 6. VA061216

Raimondiceras pflückeri (Lissón, 1904).

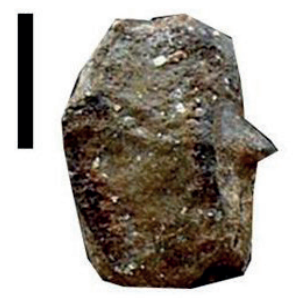

Fig. 9.VA091216.

Raimondiceras pflücker (Lissón, 1904).

Corte transversal

Escala $1 \mathrm{~cm}$ 